\title{
A Global view of the Eclipse over the Earth (GEE) in 2009 and 2012
}

\author{
T. Handa ${ }^{1}$, K. Hata ${ }^{2}$, T. Hara ${ }^{3}$, T. Horaguchi ${ }^{4}$, M. Hiramatsu ${ }^{5}$, \\ T. Arai ${ }^{6}$, Y. Sato ${ }^{7}$ and K. Ohnishi ${ }^{8}$ \\ ${ }^{1}$ Depart. Astron. \& Phys., Kagoshima Univ., Korimoto 1-21-35, Kagoshima 890-0065, Japan \\ email: handa@sci.kagoshima-u.ac.jp \\ ${ }^{2}$ Okayama Shoka University Highschool , ${ }^{3}$ Toyooka Highschool, Saitama \\ ${ }^{4}$ National Museum of Nature and Science, Tokyo, ${ }^{5}$ National Astron. Obs. of Japan \\ ${ }^{6}$ Katsushika City Museum, ${ }^{7}$ Hokkaido University, ${ }^{8}$ Nagano National College of Technology
}

A solar eclipse is one of the most popular events in astronomy. Although it is the single astronomical event, it gives different images from place to place and changes in time. It is the most important message from astronomy to the public that an apparent face depends on the viewpoint and we should get the comprehensive view.

A map made of many movies taken from many locations can be helpful to understand what happens actually and to get the comprehensive view of the eclipse. Therefore, we promoted a campaign to take synchronized sequential images of the eclipsing sun and to draw a "big picture" made from them. The campaign was done both in 1997, 2009, and 2012. In 2012 we successfully got many shots in every 5-min interval or more frequently from more than 20 locations over Japan and Taiwan.

From our archive we have made a sample movie of the annular eclipse through Taiwan and Japan, which can be watched via our web site located at

http://milkyway.sci.kagoshima-u.ac.jp/ handa/eclipse/. Combined with a CG movie watched from the space for instruction, we can make a better talk on the solar eclipse with the comprehensive view.

From our archive we will choose images enough accurate to estimate a geometrical parallax and/or moving velocity of the Moon shadow on Earth (e.g. Handa et al. 2000). Using these data, we can make a lesson for students to derive the mass of Earth.

Using the same observation skill and instruments we observed the Venus transit on this June. We coordinated synchronous observations at different locations. A pair of images from two distant sites gives geometrical parallax to estimate 1 AU. At present we have succeeded to get at least one pair from USA and Japan baseline with longer than 8000 $\mathrm{km}$ baseline. As a preliminary result we got the distance from Earth to the Sun to be about $1.6 \times 10^{8} \mathrm{~km}$. We should offer many available pairs, because redundancy is essential to check the consistency and it is very important for science.

The astronomical unit is the most basic length in astronomy and a good introduction to approach any modern astrophysics (see proceedings of IAU Symp. 289 held in Beijin General Assembly). We will make a textbook for high school students to estimate 1 AU from these images as the next step with the help of GHOU and GTTP members.

\section{Reference}

Handa T., Agata H., \& Fukushima T. 2000, Astronomical Herald, 93, 432 